\section{Quantum Effects Challenge Navier-Stokes Equation}

\section{Shantilal Gangadas Goradia*}

President, Gravity Research Institute, Inc., Shantiniketan1, 983 David Walker Drive, Tavares, FL 32778 USA

\begin{abstract}
In our previous articles, we explained various issues by using our probabilistic gravity such as constants of Nature including strong coupling, information, consciousness, entanglement, cause of Alzheimer's, spooky dark matter, multiplicity of particles, Brownian motion, synchronicity and some issues in biology etc. etc. Here, we extend it to medical and engineering issues, while addressing the Clay mathematics institute's problem about Navier-Stokes Equation on their web site.
\end{abstract}

Keywords: Alzheimers; Entanglement; Biology

\section{Introduction}

Navier-Stokes Equation is a partial differential equation for a fluid flow. Its medical and engineering significance is adequately described in [1-3], demonstrating the importance of its solution. "Turbulent flows by coughs and sneezes...have come to the forefront because of COVID 19" [2]. However, its solution is impossible. Nevertheless, the Clay Institute has sponsored a prize for its solution as below. Its website breaks it down into four problems. The prize requires one to solve any one of them. In all four, the fluid is assumed incompressible. "They are:

1. Existence and smoothness in solutions in three dimensions. Here the fluid is assumed to fill the whole of infinite space. Given any initial smooth velocity field, prove that a smooth solution to the equation exists for all positive times, coinciding with the specified initial field.

2. Existence and smoothness of solutions in the three-dimensional flat torus. The same question, but now assuming that space in flat torus-a rectangular box with opposite faces identified. This version avoids potential problems caused by the infinite domain assumed in the first version, which does not match reality and might cause bad behavior for silly reasons.

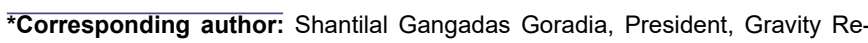
search Institute, Inc., Shantiniketan1, 983 David Walker Drive, Tavares, FL 32778 USA, E-mail: shantilalg1@juno.com

Citation: Goradia SG (2021) Quantum Effects Challenge Navier-Stokes Equation. J Alzheimers Neurodegener Dis 7: 053.

Received: June 03, 2021; Accepted: June 05, 2021; Published: June 12, 2021

Copyright: (c) 2021 Goradia SG. This is an open-access article distributed under the terms of the Creative Commons Attribution License, which permits unrestricted use, distribution, and reproduction in any medium, provided the original author and source are credited.
3. Breakdown of solutions in three dimensions. Prove that (1) is wrong. That is find an initial field for which a smooth solution does not exist for all positive times, and prove that statement.

4. Breakdown of solutions in the three dimensions flat torus. Prove that (2) is wrong. [1]"

We test our quantum gravity for the solution of the above, we touch base about the fundamental cause of meditation and other tidbits.

\section{Test of Our Probabilistic Gravity}

Our probabilistic theory [4,5], first put on the preprint achieve, implies in short that the probability of an interaction between any two particles is inversely proportional to their separation in Planck lengths i.e. one particle interacts with multiple particles, each one generating a tiny black hole, though too tiny to be noticeable to us as black holes, they can disturb the fluid flow. Each entangled particle has its own gravitational effect per [6] now backed by Feynman's 1957 view, dug out by Tim Folger of Scientific American [7]. Each Planck time has its own significance, because the natural log of the age of the universe in Planck times (10E60 Planck times) comes out admirably close to Feynman's mystical number 137 [8] within a decimal point. After numerous presentations, we placed it on a future grave yard stone.

Dr. Stewart's statement says, “....particles interact with each other [1] p 234", "This very statement gives birth to a black hole: When two nucleons interact with each other, they create a black hole per Karl Schwarzschild's impressive equation as in equation 1 of [9]. This creates a strong coupling with a known force of $10 \mathrm{E} 40 \mathrm{~g}$ which diminishes to gravity, outside an atom [9]. Existence of gravity indicates the preexistence of black holes that create gravity waves. The implicit synonymy of strong coupling and gravitation is elaborated in [10] and [11] with [11] taking a relativistic approach. Such black holes and their forces appear ON and OFF per our probabilistic (statistical) gravity [8], implying they are not continuous. The Navier-Stokes equation is based on the Newtonian law of gravity equating force to the product of mass and acceleration, which have continuity, questioning its validity at Planck scale. The self-supporting consequence of discontinuity is that it provides for the information, as claimed in [8]. Radiation of information in [3] is an alternate description of the same idea. Somehow once we made now Nobel Laureate Kip Thorne smile in GR17 Conference in Dublin, Ireland mentioning our proposal of the synonymy of nuclear force and gravitation.

The subject problem about the Navier-Stokes equation asks whether singularities can occur in initial value problems, for a fluid occupying either the whole of space or a flat torus. [1] p 225. If a singularity occurs in a finite time, the result is likely to be blown up, unless the singularity somehow unravels itself subsequently, which seems unlikely, [1] p 225. Dr. Stewart mentions on p 221, "Many different scenarios that might lead to singularities have been investigated." A black hole leads to singularity per accepted physics. Our quantum gravity has innumerable black holes therefore, singularities are their undeniable consequences at all Planck times i.e. in all initial 
value problems. Therefore, (1) and (2) are wrong, and (3) and (4) are right. It is that simple! Dirac craved for simplicity. "Analytical solutions aren't possible in the Navier-Stokes equation for turbulent flows [1]" Obviously so, the analytical solutions are devoid of innumerable and invisible tiny black holes that disturb the smoothness of the flow. There are additional substantiations.

\section{Response to Clay Institute Website}

We agree with Charles Fefferman about Navier-Stokes equation. "In his article about this problem on the Clay institute website [http:// www.claymath.org/millennium/Navier-Stokes_Equations/], Charles Fefferman writes: Standard methods (from partial differential equations) appear inadequate to settle the problem. Instead, we probably need some deep, new idea. [1] p 227" The deepness of our idea is in the following published on the subject of "Reflections" in [12] editted by Shantiniketan's resident, psychiatric and medical Dr. Kedar Berma. "Soon after learning the Newton's Law of Gravity in the high school, I asked the teacher in my childish language then, about its failure to explain the strong binding energy at the smallest particles. The teacher highly appreciated the question and replied that Einstein may have explained it. Einstein did so in 1919, but his explanation was non-quantum and was not accepted. Quantum mechanics was not developed then to explain it anyway. So in 3/1999, we found a partial quantum mechanical solution, now in our book [5] and used it to ask a question to Nobel Laureate, Dr. Weinberg as detailed in [6]. Our references in [6] imply the reflections of that question, one addressing the problem of dark matter, now at the forefront of physics as explained in (DOI: 10.17265/2159-5348/2015.05.008) [9] matching the case of Lord Shiva's Tandam Natyam." We were proud to see Lord Shiva's dance picture in The Wall Street Journal on 4/5/2016 with a figure head: CERN's Shiva, which we referred in [13]. In the words of the late scientist Carl Sagan on that dance, "It is the clearest image of the activity of God which any art or religion can boast of [14]."

The above childish question to the teacher was to verify the lower boundary condition of the inverse square law. Later we learnt that his doubt in his own inverse square formula made Newton delay its publication for over a decade until forced by the Royal Society. We deserve his blessings from Heaven.

\section{Meditation and Philosophy}

Referring Occam's razor and [15] for author's explicit view, "Particles have a quantum mouth, and a body they can stretch, and get entangled" says Dr. Zacharia P. Thundy, author of dozens of such books, who has devoted two pages with a comment: "The simplest solution needs to be the right one [16]" A nucleon can stretch out of the divisible atom and interact with other nucleons, causing a strong coupling.

Our 2020 horizon [17] hypotheses the multiplicity of soul in the Higgs field. One possibility of such a field is speculated by Dr. Weinberg in [18]. Surprisingly, Bhagwat Gita 13.1-7 repeatedly asserts such a field. We see the possibility of the fundamental cause of meditation in the multiplicity of soul (mind) in the HIGGS field in [17].

\section{Fluid Flow Fig. Resembles Quantum Foam}

Talking about four cases of fluid flows with their pictures in a figure (Figure 43) [1] p 227, it states "The complexity of the flow in images like Figure 43 drives home the difficulties that are likely to be encountered when seeking these ideas. Undaunted, mathematicians are soldering on, seeking simple principles within the apparent complexities [1] p 227" The subject Figure 43 (with a figure head: Zooms into a turbulent flow, ....) in the book [1], and also the one colorfully shown in [2] look more like a quantum foam depicted by Stephen Hawking in his book rather than one showing a smooth flow, as if it is affected by the quantum effects in the fluid, substantiating our application of quantum gravity and questioning the smoothness of the flow. It would be interesting to see similar pictures of Bermuda Triangle accidents with no known cause.

\section{Feynman's View}

"There is a physical problem that is common to many fields, that is very old, and that has not been solved. It is not the problem of finding new fundamental particles, but something left over from a long time ago---over a hundred years. Nobody in physics has really been able to analyze it mathematically satisfactorily in spite of its importance to the sister sciences. It is the analysis of circulating or turbulent fluids $[2,19]$ ". The sister sciences include medical science about blood flow through veins and aeronautical engineering. Dr. Perry adds in the first paragraph, "Evan today in the age of supercomputers, the need for understanding, modeling, and predicting aspects of turbulent flows has, if anything, increased. Reliably simulating turbulent flows still requires more theoretical advances, and Feynman's vision of "Solving the problem of turbulence" remains elusive [2]" The theoretical advance we propose is our quantum theory of gravity devoid of the need of unfound gravitons.

\section{Applicability to Medical Science}

Einstein's 1919 paper [20] concludes synonymy of nuclear force and gravity without needing the graviton. He transcended particle mechanisms anyway. In 1905, when Einstein investigated Brownian motion, there was no quantum mechanics. Considering Brownian motion as a non-zero turbulence, we need to find its fundamental quantum mechanical cause, because that is the one that gets pronounced with higher fluid velocities. Our quantum gravity [8], goes deeper into explaining the Brownian motion. Unlike cosmic black holes which are not surrounded by fluid, turbulence must be increased with high velocity fluid flow and black hole combinations, affecting the smoothness of the blood flow.

\section{String Theory}

A preoccupation with the notion that a particle cannot interact with more than one particle simultaneously denies one to explain the proven observation of multiplicity of particles. What is obligatory and therefore of primary importance in string theory is the quantum theory of gravity combined with Feynman's mystical number ALPHA. God made Nature somehow equates the cumulative effect of the sum of all coupling constant forces to the force of gravity we perceive, questioning the need for an unfound graviton missing for a century. Gravitons cannot create information needed for the consciousness that exists in life, bypassing biology/medical science from the theory of everything. The wonderful author of the book, "GOD EQUATION" says "WANTED: THE GRAVITON [3]". There already is a God Particle (Higgs Boson) in the Higgs Field derived from its own equation. String theory may someday prove the roundness of the earth, but if gravity is the cumulative effect of the coupling constants of Nature as implied by our quantum theory of gravity, graviton will never be found even if we spend another century to find it. The standard model has no graviton for a reason. String 
theory is a word by Ed Witten, the word is not written (accepted). The need for the graviton is based on the assumption that gravity is a fundamental interaction. Such assumption implies that Nature created the coupling constants for nothing. Nature creates nothing useless per Nobel Laureate Dr. Tagore; we agree. The string theory assumes permanent unverifiable dimensions. The probabilistic string like connections between elementary particles, implicit in our theory, are observed in quantum tunneling as sketched in $[5,21]$.

\section{Conclusion}

The reason why the Navier-Stokes equation cannot have a solution is that its derivation uses classical (Newtonian) continuum gravity which we say is affected on the cumulative effect of the manifestation of the forces of black holes generated by particle interactions which are fundamentally non-continuous point like sources at Planck scales per our quantum theory of gravity. In other words, the fluid flows are affected by our promising quantum theory of gravity which is devoid of gravitons. The new horizon implies that the quest for TOE in [3] is based on probabilistic mathematics; not deterministic one, assumed in [3]. Our probabilistic approach can substitute for the others not so successful.

\section{References}

1. Stewart I (2013) Visions of Infinity, The Great Mathematical Problems. Basic Books.

2. Johnson PL (2021) The squeezes, stretches, and whirls of turbulance, Fluid turbulence generates a chaotic soup of strongly nonlinear features that are hard to mathematically describe and model. A recent theoretical simplification brings fresh insight. Physics Today.

3. Kaku M (2021) The God Equation: The Quest for a Theory of Everything.

4. Goradia S (2012) Newtonian gravity in natural units. Journal of Physical Science and Application 2: 265-268.

5. Goradia S (2011) Quantum Consciousness-The Road to Reality. Journal of Life Sciences 10: 269-272.

6. Goradia S (2019) The Quantum Theory of Entanglement and Alzheimer's. J Alzheimer's Neurodegener Dis 5: 023.
7. Folger T (2019) Quantum Gravity in the lab. Scientific American.

8. Goradia S (2015) Decoding the information of life. Journal of Physical Science and Application 5: 191-195.

9. Goradia S (2015) Dark Matter from Our Probabilistic Gravity. Journal of Physical Science and Application 5: 373-376.

10. Goradia S (2006) Why is Gravity so Weak? Journal of Nuclear Radiations and Physics 1: 107-117.

11. Shrivastava SK (1928) Aspects of Gravitational Interactions, Nova Science Publishers: Hauppauge, USA, p91.

12. Goradia S (2020) Tandam Natyam and Bhagwat Gita. Lamp, Shantiniketan Community Magazine, Tavares, FL, Edited by Dr. Kedar Verma.

13. Goradia S (2017) The Emporer's mind in a nut shell. J of Life Sciences 11: $249-253$.

14. Somal B (2015) World's Intellectual on Bharat (India) p36.

15. Goradia S (2006) My Focus on the Quantum Source of Gravity. Frontiers of Fundamental Physics 37-44.

16. Thundy ZP (2018) God: Etymology, Patrology/Matrology, Anthropology--A lede -All, Zacharia Thundy, Professor Emeritus, Northern Michigan University, Tomas Books.

17. Goradia S (2020) The Scientific Search for the Soul in the Higgs Field, J Alzheimer's Neurodegener Dis 6: 049.

18. Baggott J (2013) Higgs: The Invention and Discovery of the God Particle with a forward by Steven Weinberg.

19. Feynman RP, Leighton RP, Sands M (1964) The Feynman Lectures on Physics. Addison-Wesley 1: 3-7.

20. Einstein A (1952) Do Gravitational Fields Play an Essential Part in the structure of the Elementary Particles of Matter? In: The Principle of Relativity, Dover Publications: Mineola, USA, pp. 189-198.

21. Goradia S (2016) Quantum Consciousness-The Road to Reality. Journal of Life Sciences 10: 1-4. 


\section{H}

Advances In Industrial Biotechnology | ISSN: 2639-5665

Advances In Microbiology Research | ISSN: 2689-694X

Archives Of Surgery And Surgical Education | ISSN: 2689-3126

Archives Of Urology

Archives Of Zoological Studies | ISSN: 2640-7779

Current Trends Medical And Biological Engineering

International Journal Of Case Reports And Therapeutic Studies | ISSN: 2689-310X

Journal Of Addiction \& Addictive Disorders | ISSN: 2578-7276

Journal Of Agronomy \& Agricultural Science | ISSN: 2689-8292

Journal Of AIDS Clinical Research \& STDs | ISSN: 2572-7370

Journal Of Alcoholism Drug Abuse \& Substance Dependence | ISSN: 2572-9594

Journal Of Allergy Disorders \& Therapy | ISSN: 2470-749X

Journal Of Alternative Complementary \& Integrative Medicine | ISSN: 2470-7562

Journal Of Alzheimers \& Neurodegenerative Diseases | ISSN: 2572-9608

Journal Of Anesthesia \& Clinical Care | ISSN: 2378-8879

Journal Of Angiology \& Vascular Surgery | ISSN: 2572-7397

Journal Of Animal Research \& Veterinary Science | ISSN: 2639-375

Journal Of Aquaculture \& Fisheries | ISSN: 2576-5523

Journal Of Atmospheric \& Earth Sciences | ISSN: 2689-8780

Journal Of Biotech Research \& Biochemistry

Journal Of Brain \& Neuroscience Research

Journal Of Cancer Biology \& Treatment | ISSN: 2470-7546

Journal Of Cardiology Study \& Research | ISSN: 2640-768X

Journal Of Cell Biology \& Cell Metabolism | ISSN: 2381-1943

Journal Of Clinical Dermatology \& Therapy | ISSN: 2378-8771

Journal Of Clinical Immunology \& Immunotherapy | ISSN: 2378-8844

Journal Of Clinical Studies \& Medical Case Reports | ISSN: 2378-880

Journal Of Community Medicine \& Public Health Care | ISSN: 2381-1978

Journal Of Cytology \& Tissue Biology | ISSN: 2378-9107

Journal Of Dairy Research \& Technology | ISSN: 2688-9315

Journal Of Dentistry Oral Health \& Cosmesis | ISSN: 2473-6783

Journal Of Diabetes \& Metabolic Disorders | ISSN: 2381-201X

Journal Of Emergency Medicine Trauma \& Surgical Care | ISSN: 2378-8798

Journal Of Environmental Science Current Research | ISSN: 2643-5020

Journal Of Food Science \& Nutrition | ISSN: 2470-1076

Journal Of Forensic Legal \& Investigative Sciences | ISSN: 2473-733X

Journal Of Gastroenterology \& Hepatology Research | ISSN: 2574-2566
Journal Of Genetics \& Genomic Sciences | ISSN: 2574-2485

Journal Of Gerontology \& Geriatric Medicine | ISSN: 2381-8662

Journal Of Hematology Blood Transfusion \& Disorders | ISSN: 2572-2999

Journal Of Hospice \& Palliative Medical Care

Journal Of Human Endocrinology | ISSN: 2572-9640

Journal Of Infectious \& Non Infectious Diseases | ISSN: 2381-8654

Journal Of Internal Medicine \& Primary Healthcare | ISSN: 2574-2493

Journal Of Light \& Laser Current Trends

Journal Of Medicine Study \& Research | ISSN: 2639-5657

Journal Of Modern Chemical Sciences

Journal Of Nanotechnology Nanomedicine \& Nanobiotechnology | ISSN: 2381-2044

Journal Of Neonatology \& Clinical Pediatrics | ISSN: 2378-878X

Journal Of Nephrology \& Renal Therapy | ISSN: 2473-7313

Journal Of Non Invasive Vascular Investigation | ISSN: 2572-7400

Journal Of Nuclear Medicine Radiology \& Radiation Therapy | ISSN: 2572-7419

Journal Of Obesity \& Weight Loss | ISSN: 2473-7372

Journal Of Ophthalmology \& Clinical Research | ISSN: 2378-8887

Journal Of Orthopedic Research \& Physiotherapy | ISSN: 2381-2052

Journal Of Otolaryngology Head \& Neck Surgery | ISSN: 2573-010X

Journal Of Pathology Clinical \& Medical Research

Journal Of Pharmacology Pharmaceutics \& Pharmacovigilance | ISSN: 2639-5649

Journal Of Physical Medicine Rehabilitation \& Disabilities | ISSN: 2381-8670

Journal Of Plant Science Current Research | ISSN: 2639-3743

Journal Of Practical \& Professional Nursing | ISSN: 2639-568

Journal Of Protein Research \& Bioinformatics

Journal Of Psychiatry Depression \& Anxiety | ISSN: 2573-0150

Journal Of Pulmonary Medicine \& Respiratory Research | ISSN: 2573-0177

Journal Of Reproductive Medicine Gynaecology \& Obstetrics | ISSN: 2574-2574

Journal Of Stem Cells Research Development \& Therapy | ISSN: 2381-2060

Journal Of Surgery Current Trends \& Innovations | ISSN: 2578-7284

Journal Of Toxicology Current Research | ISSN: 2639-3735

Journal Of Translational Science And Research

Journal Of Vaccines Research \& Vaccination | ISSN: 2573-0193

Journal Of Virology \& Antivirals

Sports Medicine And Injury Care Journal | ISSN: 2689-8829

Trends In Anatomy \& Physiology | ISSN: 2640-7752

Submit Your Manuscript: https://www.heraldopenaccess.us/submit-manuscript 